\title{
P-Akt/miR-200 signaling regulates epithelial-mesenchymal transition, migration and invasion in circulating gastric tumor cells
}

\author{
DANDAN YUAN ${ }^{1,2}$, HONGWEI XIA ${ }^{2}$, YUCHEN ZHANG $^{2}$, LIANG CHEN $^{2}$, WEIBING LENG $^{1,2}$, TIE CHEN $^{3}$, \\ QINGJUAN CHEN ${ }^{1,2}$, QIULIN TANG ${ }^{2}$, XIANMING MO $^{3}$, MING LIU $^{1}$ and FENG BI ${ }^{1,2}$ \\ ${ }^{1}$ Department of Medical Oncology, ${ }^{2}$ Laboratory of Signal Transduction and Molecular Target Therapy and \\ ${ }^{3}$ Laboratory of Stem Cell Biology, West China Hospital, Sichuan University, Chengdu 610041, P.R. China
}

Received June 10, 2014; Accepted July 25, 2014

DOI: 10.3892/ijo.2014.2644

\begin{abstract}
Both circulating tumor cells (CTCs) and epithelialmesenchymal transition (EMT) play an important role in invasion, migration and chemoresistant in tumor development. This study aimed to detect whether EMT occurred in human gastric CTCs and to explore the mechanism of EMT in human gastric CTCs. We analysed epithelial markers (pan-CK, E-cadherin), mesenchymal markers (N-cadherin, vimentin) EMT related miR-200s, and Akt in gastric CTCs. The impact of miR-200s on EMT, migration and invasion in CTCs was tested. We found that epithelial markers pan-CK, E-cadherin were decreased, and mesenchymal markers $\mathrm{N}$-cadherin, vimentin were overexpressed in gastric CTCs. Expression of EMT related transcriptors, snail1, zeb1, twist1, were reversely correlated with miR-200s, and were positively correlated with phospho-Akt. Upregulated of miR-200s downregulated twist1 and zeb1 mRNA expression, and resulted in the supression of EMT, and impaired migration and invasion in gastric CTCs. Inhibition of p-Akt led to upregulation of miR-200s. In conclusion, gastric CTCs exhibited remarkable EMT process, and p-Akt/miR-200s signaling regulates EMT, migration and invasion in gastric CTCs.
\end{abstract}

\section{Introduction}

According to statistics of 2012 International Agency for Research on Cancer (IARC), almost one million new cases

Correspondence to: Professor Feng Bi or Dr Ming Liu, Department of Medical Oncology, West China Hospital, Sichuan University, Chengdu 610041, P.R. China

E-mail: bifeng@medmail.com.cn

E-mail: mingliu721@aliyun.com

Abbreviations: CTCs, circulating tumor cells; EMT, epithelialmesenchymal transition; miR, microRNA

Key words: circulating tumor cells, epithelial-mesenchymal transition, miR-200s, p-Akt of gastric cancer were estimated to have occurred in 2012 (952,000 cases, $6.8 \%$ of the total), making it the fifth most common malignancy in the world, after cancers of the lung, breast, colorectum and prostate. More than $70 \%$ of cases $(677,000$ cases) occur in developing countries $(456,000$ in men, 221,000 in women), and half the world total occurs in Eastern Asia (mainly in China). Gastric cancer is the third leading cause of cancer death in both genders worldwide (723,000 deaths, $8.8 \%$ of the total), and the third leading cause of cancer death in both males and females in China (1). The poor prognosis of gastric cancer is due to its metastasis and relapse. Metastasis, as a result of dissemination and growth of cancer cells, represents the most common cause of death in cancer patients. Therefore, it is necessary to characterize the molecular mechanism of gastric cancer metastasis.

Circulating tumor cells (CTCs) are cells that leave the primary tumor and circulate in the periphery blood. CTCs are both prognostic and predictive marker for cancer patients (2-4). Previous studies also demonstrated that CTCs could help clinicians learn more about tumor biological behavior, such as, chemo-sensitivity or resistance and metastatic ability, and CTCs could provide for screening for adjuvant therapy patients, and tumor pharmacokinetics, and provide new targets for treatment (5). It is reported that CTCs participated in the initial process of metastasis (6). Thus, CTCs may have a potential utility as less invasive than standard biopsies to further understand the mechanism of metastasis.

Epithelial-mesenchymal transition (EMT) plays an important role in tumor cell invasion and metastasis (7). EMT is a complex process that refers to the transformation of epithelial cells to mesenchymal cells, in which the polarity of epithelial cells is lost, accompanied with enhanced migration and invasion (8). The characteristics of EMT is the loss of expression of epithelial cell markers (E-cadherin) and overexpression of mesenchymal cell markers (e.g., $\alpha$-smooth muscle actin protein, $\alpha$-SMA, N-cadherin, vimentin) (8-10). Recently, $\mathrm{Yu}$ et al reported that circulating breast tumor cells exhibited dynamic changes in epithelial and mesenchymal characteristics during treatment of breast cancer patients (11). In addition, miR-200b has been reported to inhibit EMT in prostate cancer cells (12). Virtakoivu et al found that inhibited Akt 2 could 
induce overexpression of miR-200s and then regulated invasion and migration of prostate cancer cells (13). Our previous study confirmed that gastric CTCs had stronger capacity of migration, invasion, metastasis, and radiation-resistance than human gastric cancer cell lines SGC-7901 and MKN-45. Thus, this study aimed to detect whether EMT occurred in gastric CTCs and participated in the process of human gastric cancer metastasis, and then to find the mechanism of EMT in human gastric CTCs.

\section{Materials and methods}

Cell lines. GES, SGC-7901, MKN-45 cell lines were purchased from Chinese Academy of Medical Sciences Cancer Cell Bank (Beijing, China). The human gastric circulating tumor cells (CTC-105, CTC-141, CTC-1, CTC-12) used in this study were previously established by $\mathrm{CD} 44^{+} / \mathrm{CD} 45^{-}$isolation. Cells were cultured in Dulbecco's modified Eagle's medium (DMEM; Invitrogen, Carlsbad, CA, USA) supplemented with $10 \%$ fetal bovine serum (FBS; Hyclone, Beijing, USA). All of the cell lines were maintained in a humidified atmosphere containing $5 \% \mathrm{CO}_{2}$.

Western blot analysis. Protein extracts were resolved by 12\% SDS-PAGE electrophoresis (Bio-Rad, USA), and transferred to PVDF membranes (Millipore, MA, USA), and probed with antibodies against human $\mathrm{N}$-cadherin (1:4,000, Epitomics, USA), E-cadherin (1:1,000, Abcam, USA), vimentin (1:4,000, Epitomics), Akt (1:1,000, CST, USA), phospho-Akt (473) (1:4,000, Epitomics), anti-zeb1 antibody (1:1,000, Abcam) or GAPDH (1:1,000, Sigma, USA). Fluorescence-conjugated anti-mouse or rabbit IgG $(1: 10,000$, Sigma) were used as the secondary antibodies, and the antigen-antibody reactions were visualized using LI-COR Odyssey Infrared Imaging System (USA). Triple tests were replicated.

Triple staining of immunofluorescence analysis. Cells were fixed with $4 \%$ paraformaldehyde for 20 min and blocked in 1X PBS (pH 7.4) solution with $1 \%$ BSA. The anti-N-cadherin (1:400, Epitomics), anti-E-cadherin (1:300, Abcam), antivimentin (1:400, Epitomics), or anti-pan-CK (1:300, Abcam), were added and incubated overnight at $4^{\circ} \mathrm{C}$ in a humidified box. After washing, the fluorescent secondary antibody (Epitomics) was added at a dilution of 1:400 and incubated for 1.5 h. The cells were then washed three times with PBS, and counterstained with DAPI (Sigma) for $2 \mathrm{~min}$. Fluorescence was analyzed using a fluorescent microscope (Zeiss, Germany). Triple tests were replicated.

Quantitative real-time PCR. Total RNA was isolated with the TRIzol reagent (Invitrogen, USA) from cells according to the manufacturer's instructions. Single-strand cDNA was synthesized from $1 \mu \mathrm{g}$ of total RNA by reverse transcription according to the manufacturer's instructions (Takara, China). Single-strand cDNA for miRNA was synthesized by reverse transcription using miRNA cDNA kit according to the manufacturer's instructions (CW Biotech, China). Quantitative real-time PCR was used to measure the mRNA levels of E-cadherin, N-cadherin, vimentin, snail1, twist1, zeb1 and miRNA levels of miR-200a, b and c in gastric circu-
Table I. Primer sequences of related genes.

\begin{tabular}{|c|c|}
\hline Gene & Primers \\
\hline \multirow[t]{2}{*}{ E-cadherin } & Forward: 5'-CGAGAGCTACACGTTCACGG-3' \\
\hline & Reverse: 5'-GGGTGTCGAGGGAAAAATAGG-3' \\
\hline \multirow[t]{2}{*}{ N-cadherin } & Forward: 5'-AGCCAACCTTAACTGAGGAGT-3' \\
\hline & Reverse: 5'-GGCAAGTTGATTGGAGGGATG-3' \\
\hline \multirow[t]{2}{*}{ Vimentin } & Forward: 5'-GACGCCATCAACACCGAGTT-3' \\
\hline & Reverse: 5'-CTTTGTCGTTGGTTAGCTGGT-3' \\
\hline \multirow[t]{2}{*}{ snail1 } & Forward: 5'-TCGGAAGCCTAACTACAGCGA-3' \\
\hline & Reverse: 5'-AGATGAGCATTGGCAGCGAG-3' \\
\hline \multirow[t]{2}{*}{ twist1 } & Forward: 5'-GTCCGCAGTCTTACGAGGAG-3' \\
\hline & Reverse: 5'-GCTTGAGGGTCTGAATCTTGCT-3' \\
\hline \multirow[t]{2}{*}{ zeb1 } & Forward: 5'-TTACACCTTTGCATACAGAACCC-3 \\
\hline & Reverse: 5'-TTTACGATTACACCCAGACTGC-3' \\
\hline \multirow[t]{2}{*}{ GADPH } & Forward: 5'-CTGCACCACCAACTGCTTAG-3' \\
\hline & Reverse: 5'-TGAAGTCAGAGGAGACCACC-3' \\
\hline U6 & miRNA qPCR Primer Set (RiboBio, MQP-0201) \\
\hline miR-200a & miRNA qPCR Primer Set (Tiangen, CD201-0022) \\
\hline $\operatorname{miR}-200 b$ & miRNA qPCR Primer Set (Tiangen, CD201-0023) \\
\hline $\operatorname{miR}-200 \mathrm{c}$ & miRNA qPCR Primer Set (Tiangen, CD201-0024) \\
\hline
\end{tabular}

lating tumor cells and gastric cancer cell lines. Quantitative PCR was performed on Bio-Rad CFX manager (USA). Amplification was carried out in a $20-\mu 1$ volume in triplicate for 40 cycles and the product was detected using SYBR Green fluorochrome. The geometric average $\mathrm{Ct}$ value was used to calculate relative expression of the above genes using the method $2^{-\Delta \Delta C T}$. U6 and GADPH were used as endogenous control. Triple tests were replicated. The primers were synthesized by Genwiz Co. (Suzhou, China). The primers are listed in Table I).

Migration and invasion assay. Cell migration and invasion assays were performed as follows. For invasion assay, $1 \times 10^{4}$ cells were seeded on an $8-\mu \mathrm{m}$-pore size Transwell insert (BD, USA) coated with extracellular matrix (ECM) (1:4) (BD), while in the migration assay ECM was not used. After $48 \mathrm{~h}$ of incubation at $37^{\circ} \mathrm{C}$ and $5 \% \mathrm{CO}_{2}$, cells adherent to the upper surface of the filter were removed. Cells were stained with hematoxylin-eosin, and the number of cells on the bottom were counted under a microscope. Triple tests were replicated.

Hsa-miR-200s mimic transfection. Cells $\left(20 \times 10^{4}\right)$ were seeded in 6-well plates and grown to 50-60\% confluence. Human hsa-miR-200b and c (RiboBio, Guangzhou, China) or its negative control (RiboBio, Guangzhou, China) was directly transfected into circulating gastric tumor cells in free of serum Opti-MEM (Invitrogen) at a final concentration of $50 \mathrm{nmol}$, according to the manufacturer's protocol. 

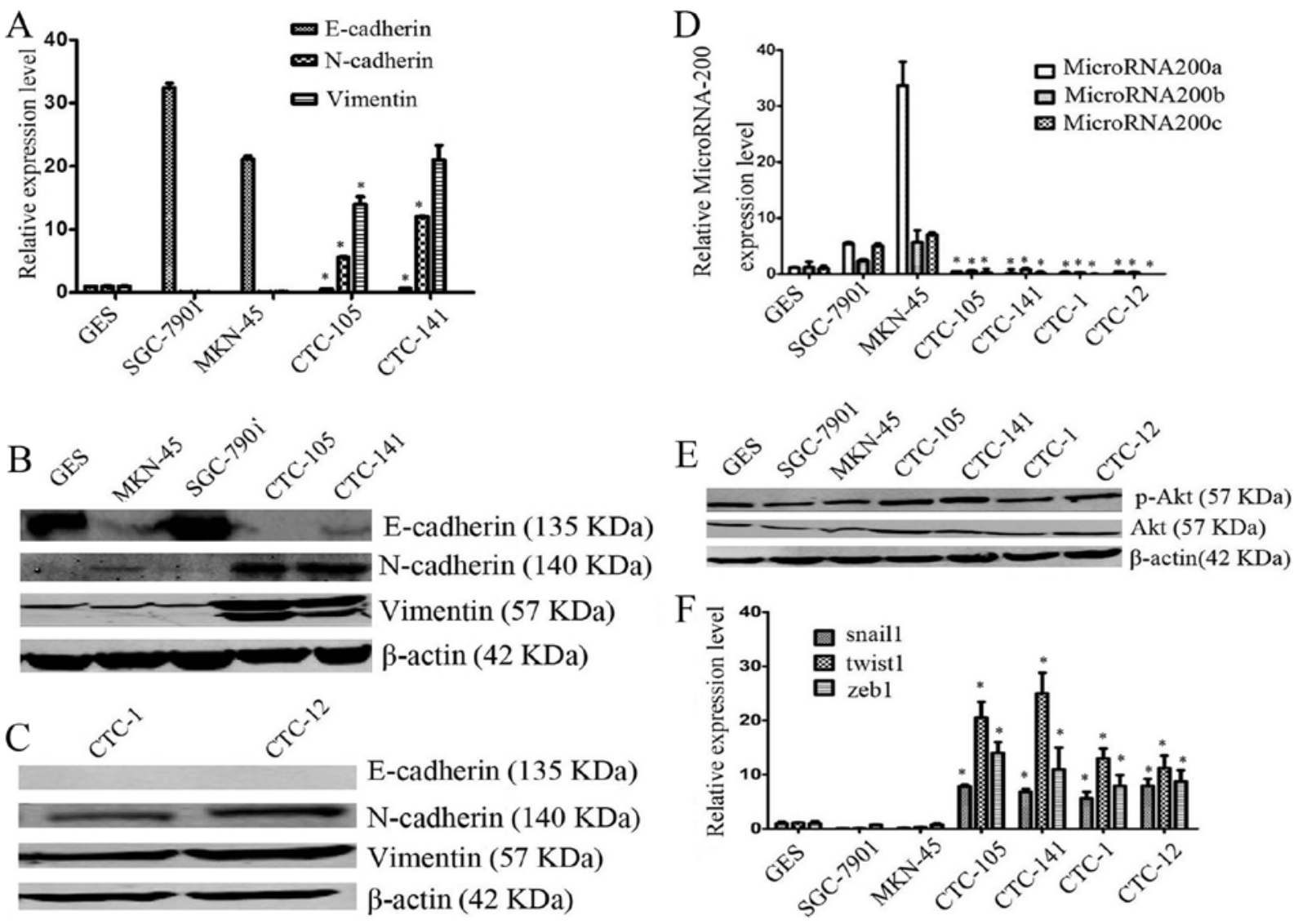

Figure 1. EMT related markers were detected in gastric CTCs and gastric cancer cell lines. (A) EMT related markers were detected in gastric CTCs and gastric cancer cell lines by quantitative real-time PCR, relative expression of the genes was calculated using the method $2^{-\Delta \Delta C T}$. (B) EMT related markers were tested in gastric CTCs and gastric cancer cell lines by western blotting. (C) Proteins were detected in two gastric CTCs by western blotting. (D) MicroRNA-200s were detected in gastric CTCs and gastric cancer cell lines by quantitative real-time PCR. (E) Total Akt and phosphor-Akt protein levels were detected in gastric CTCs and gastric cancer cell lines by western blotting. (F) EMT related transcription factors were detected in gastric CTCs and gastric cancer cell lines by quantitative real-time PCR. GES, the normal gastric mucosa epithelial cells, were used as control for quantification. Data are presented as means \pm SD from three independent experiments, one of three similar experiments is shown, ${ }^{\mathrm{P}}<0.05$, vs SGC-7901 and MKN-45.

Statistical analysis. The quantitative data are presented as mean values \pm SD from three independent experiments. One-way analysis of variance (ANOVA) was used to analyze differences among groups. In addition, the LSD multiple comparison test was used to identify differences among means of two different groups. Test level of $\alpha$ was 0.05 , P-values $<0.05$ were considered statistically significant.

\section{Results}

Gastric CTCs exhibit remarkable EMT process. To examine the differences of EMT phenotype between gastric circulating tumor cells and gastric cancer cell lines SGC-7901 and MKN-45, the mRNA and protein levels of EMT markers were measured. The relative mRNA levels of mesenchymal markers $\mathrm{N}$-cadherin, and vimentin were significantly overexpressed in circulating gastric tumor cells, compared with gastric cancer cell lines $\left({ }^{*} \mathrm{P}<0.05\right.$ for all comparation, Fig. 1A), whereas the relative mRNA level of E-cadherin was significantly decreased ( $\mathrm{P}<0.05$ for all comparisons, Fig. 1A), as assessed by real-time PCR assay. The results from western blot analysis demonstrated that the relative protein levels of $\mathrm{N}$-cadherin, and vimentin were overexpressed (Fig. 1B and C), whereas expression of E-cadherin was low in the circulating gastric tumor cells, as compared with gastric cancer cell lines, SGC-7901and MKN-45 (Fig. 1B and C). These results indicated that the expression of mesenchymal biomarkers was elevated, while expression of epithelial biomarkers was decreased, further studies of triple staining immunofluorescence suggested that, compared with gastric cancer cell lines, CTCs highly expressed the mesenchymal biomarkers vimentin and $\mathrm{N}$-cadherin, but lowly or weakly expressed epithelial biomarkers E-cadherin and pan-CK (Figs. 2 and 3).

Expression of EMT related transcriptors was reversely correlated with miR-200s and was positively correlated with Akt kinases activation in gastric CTCs. To investigate the role of miR-200s, a family of EMT-associated miRNAs, in human gastric CTCs, the relative expression of miR-200s were examined by quantitative real-time PCR assay. As expected, the relative expression of miR-200a, b and c were all significantly decreased in human gastric circulating tumor cells as compared with SGC-7901 and MKN-45 cells $(\mathrm{P}<0.05$ for all comparisons, Fig. 1D), which may suggested that miR-200s were involved in the process of EMT. To evaluate whether PI3K and Akt kinases signaling pathway is activated to participate in the process of EMT in gastric CTCs western blotting was performed to detect expression of both Akt and p-Akt 

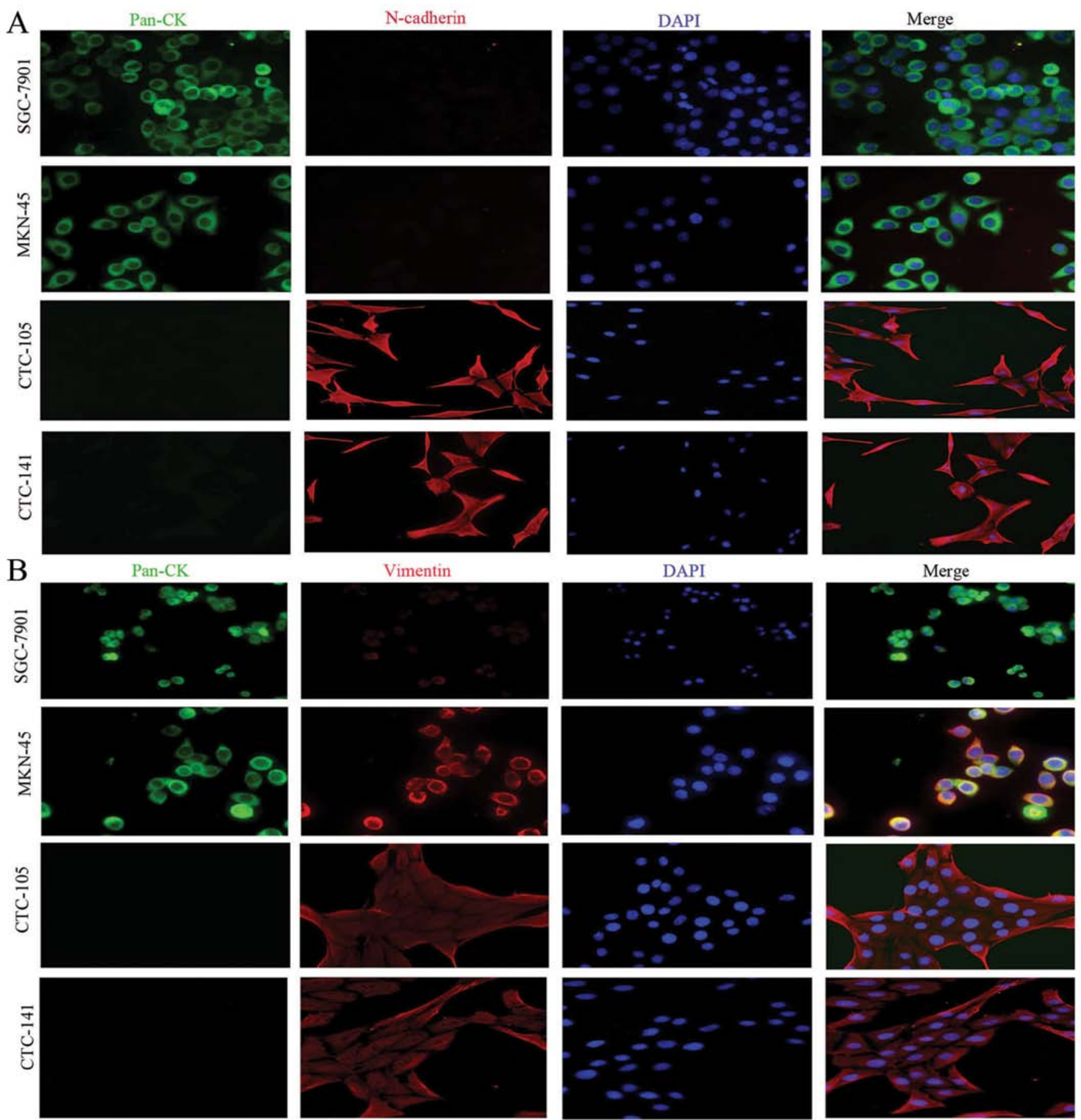

Figure 2. Epithelial marker pan-CK and mesenchymal marker vimentin and N-cadherin were detected in gastric CTCs and gastric cancer cell lines by triple immunofluorescent staining (x40). (A) pan-CK (green)/N-cadherin (in red)/DAPI (in blue) were co-stained in gastric CTCs and gastric cancer cell lines. (B) pan-CK (green)/vimentin (red)/DAPI (blue) were co-stained in gastric CTCs and gastric cancer cell lines. One of three similar experiments is shown.

(s473). It was observed that expression of both Akt and p-Akt (s473), especially p-Akt (s473), were activated in gastric CTCs (Fig. 1E). Expression of EMT-related transcription factor snail1, twist1, zeb1 was significantly overexpressed in gastric CTCs, compared with gastric cancer cell lines $(\mathrm{P}<0.05$ for all comparation, Fig. 1F). Based on the above, expression of EMT related transcriptors was reversely correlated with miR-200s and was positively correlated with Akt kinase activation in gastric CTCs.
miR-200b and c promoted E-cadherin expression and decreased twistl, zebl expression in gastric CTCs. In order to investigate the impact of miR-200b and $c$ on human gastric CTCs, human hsa-miR-200b and c or negative control miRNA were transfected into human gastric CTCs. After transfection, triple staining immunofluorescence assays were performed. Ectopic expression of miR-200b and c increased E-cadherin expression in gastric CTCs (Fig. 4). In addition, compared with negative control miRNA, ectopic expression 

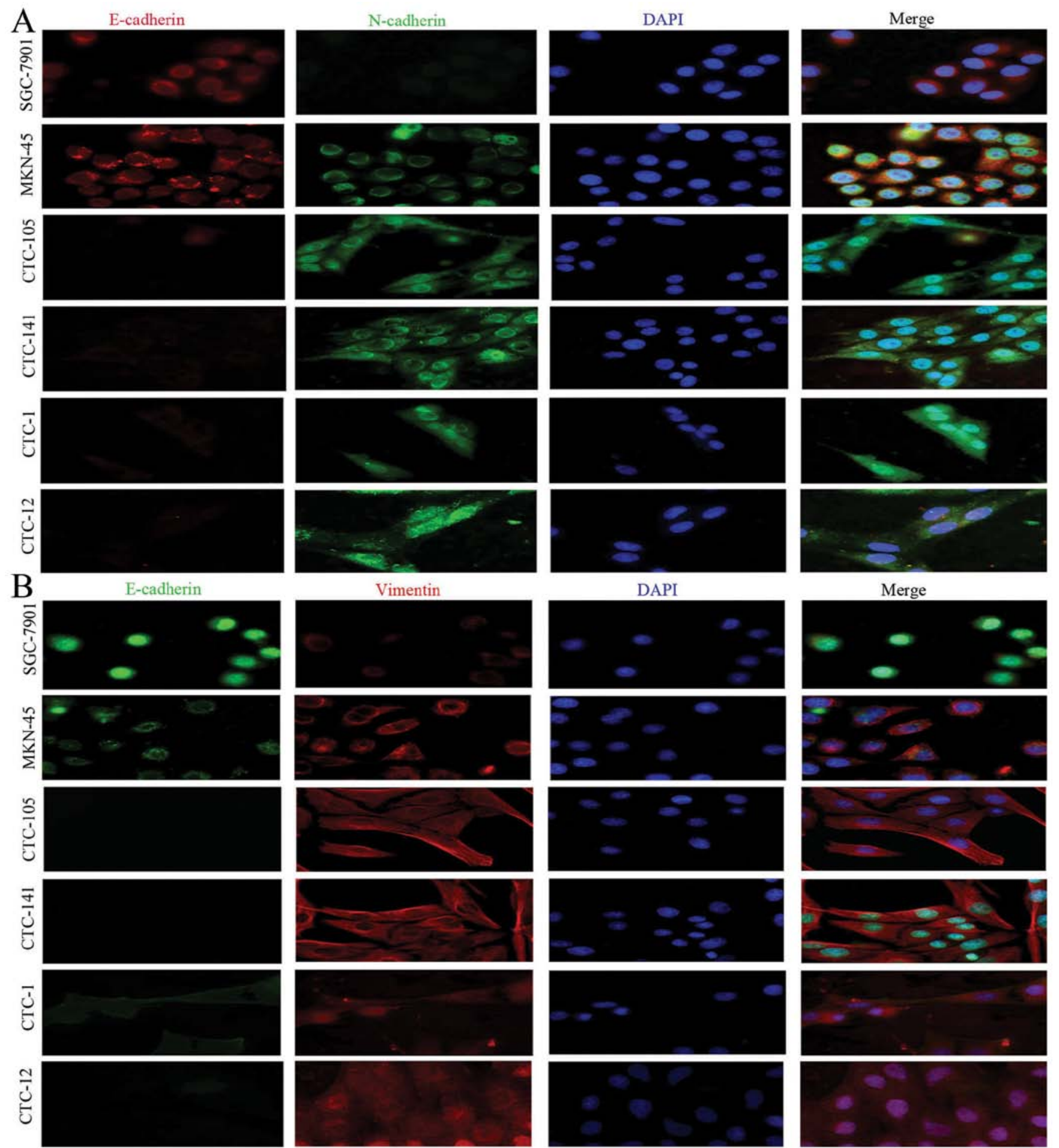

Figure 3. Triple immunofluorescent staining of epithelial marker E-cadherin and mesenchymal marker vimentin, N-cadherin in gastric CTCs and gastric cancer cell lines (x63). (A) E-cadherin (red)/N-cadherin (green)/DAPI (blue) were co-stained in gastric CTCs and gastric cancer cell lines. (B) E-cadherin (green)/vimentin (red)/DAPI (blue) were co-stained in gastric CTCs and gastric cancer cell lines. One of three similar experiments is shown.

of miR-200b or c decreased mRNA expression of snail1, twist1, zeb1 by 7.6-15.6, 2-3 and 1.58-2.3 times, respectively, in CTC-105 cells (P-values were all $<0.05$, Fig. 5A). mRNA expression of twist1, zeb1 in CTC-141 was downregulated by 1.5-2 and 1.3-1.5 times, respectively, after transfection of miR-200b or c (P-values were all $<0.05$, Fig. 5A), while snaill expression tended to increase, no significant difference was observed ( $\mathrm{P}>0.05$, Fig. $5 \mathrm{~A})$. Consistent with the results of real-time PCR, western blotting demonstrated that ectopic expression of miR-200b and c decreased the expression of zeb1 protein (Fig. 5B). Overall, miR-200b and c promoted E-cadherin expression and decreased twist1, zeb1 expression in gastric CTCs.

miR-200b and c inhibited migration and invasion in gastric CTCs. miR-200b and c are known to play key roles in the 
A

E-cadherin

$\mathrm{N}$-cadherin
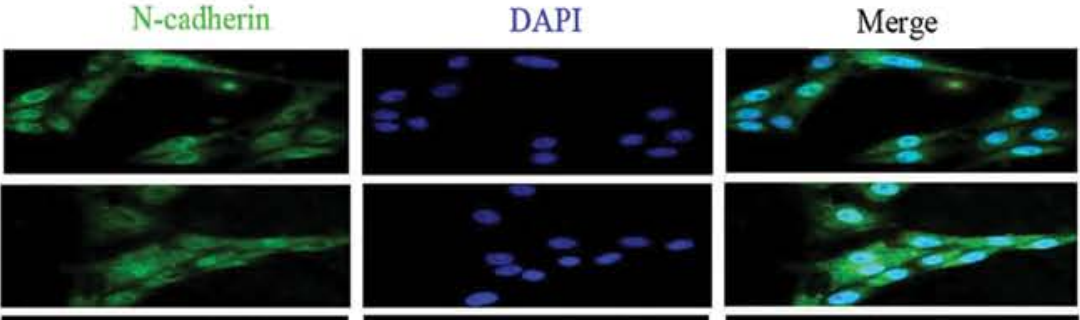

U
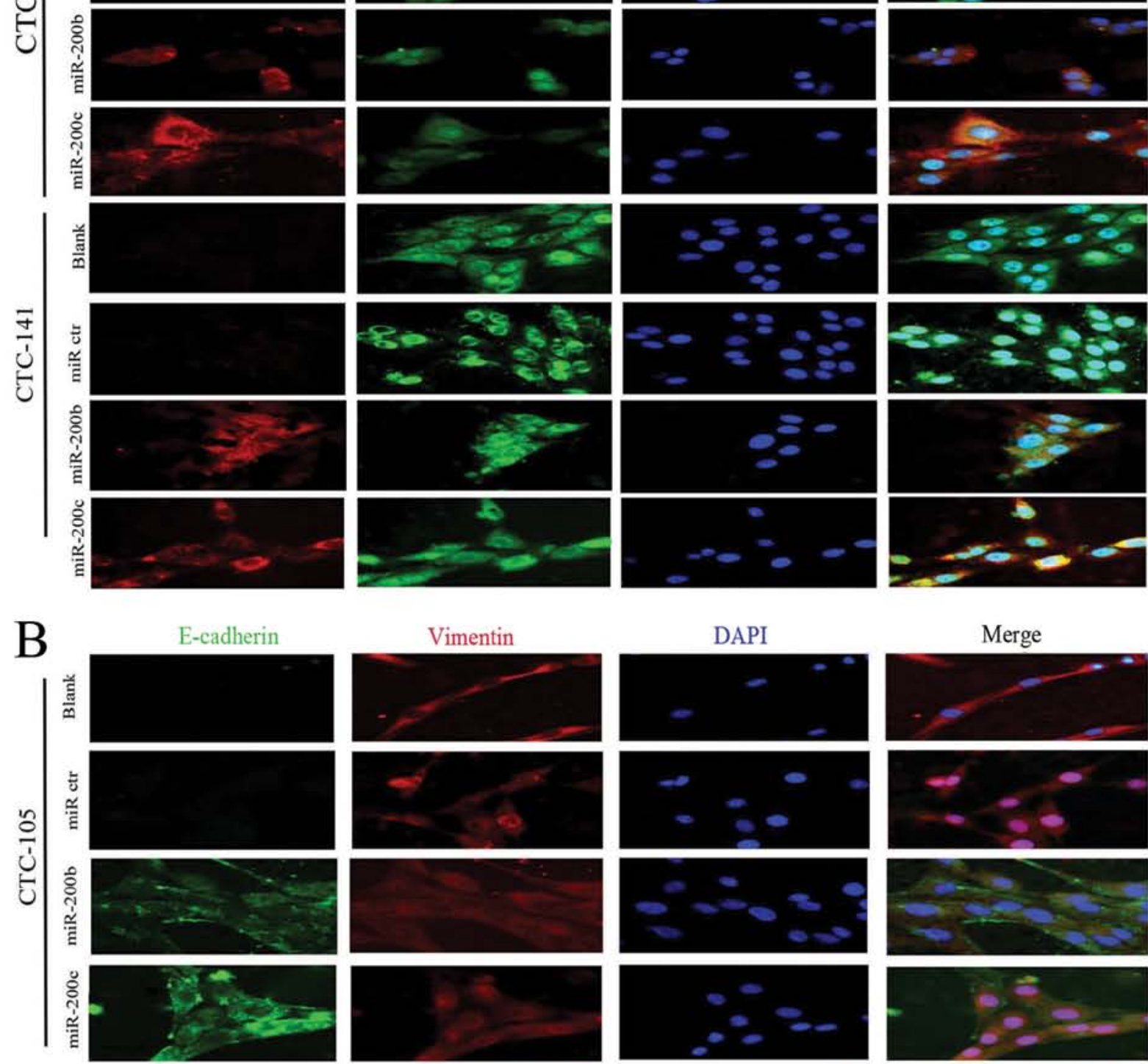

Vimentin

DAPI
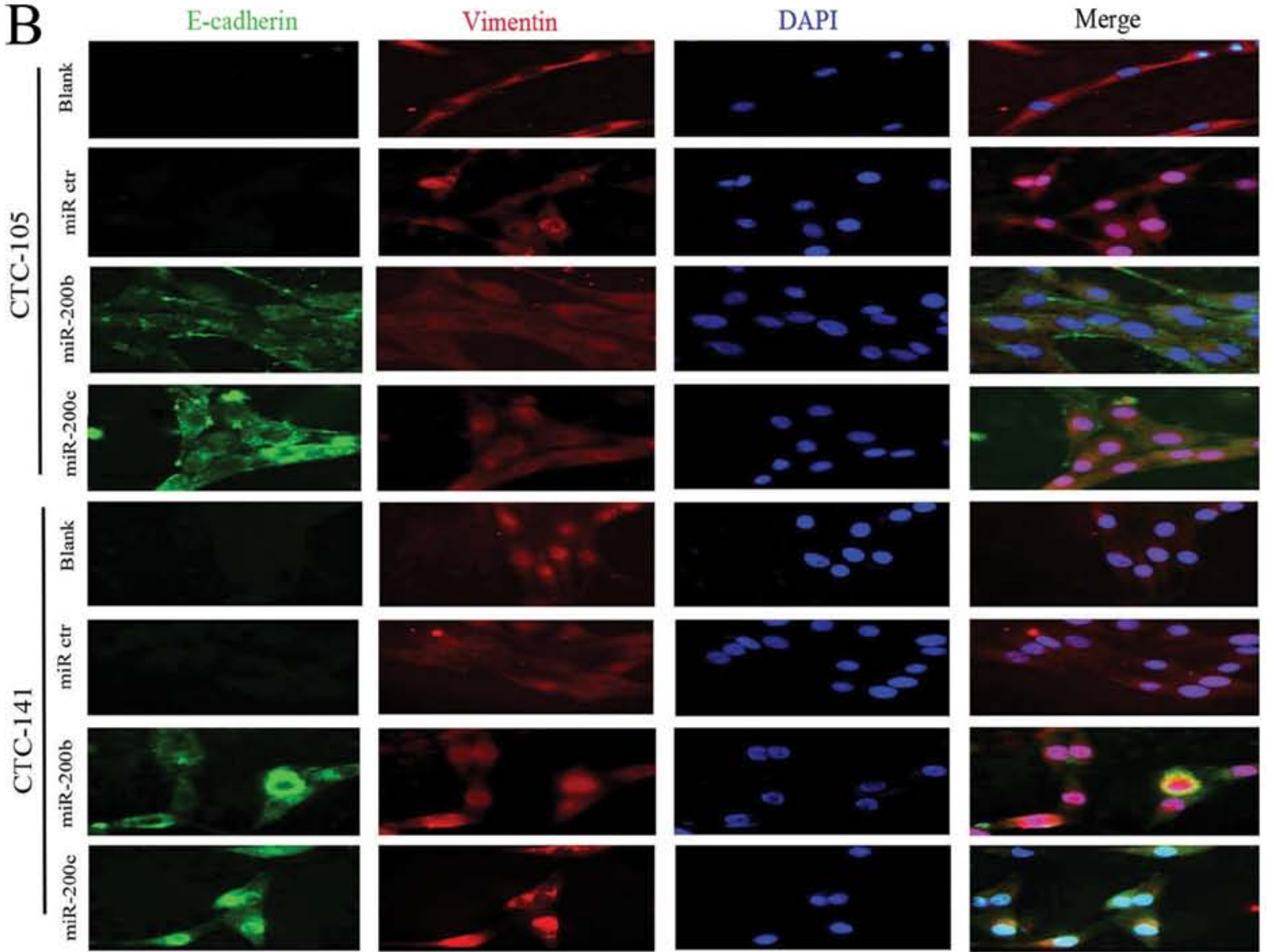

Figure 4. E-cadherin, N-cadherin and vimentin were stained after transfection of miR-200s into gastric CTCs by triple immunofluorescent staining (x63). (A) E-cadherin (red)/N-cadherin (green)/DAPI (blue) were co-stained in gastric CTCs. (B) E-cadherin (green)/vimentin (red)/DAPI (blue) were co-stained in gastric CTCs. One of three similar experiments was shown. Blank, CTC was not transfected; miR ctr, CTC trasfected with negative control miRNA. 
A

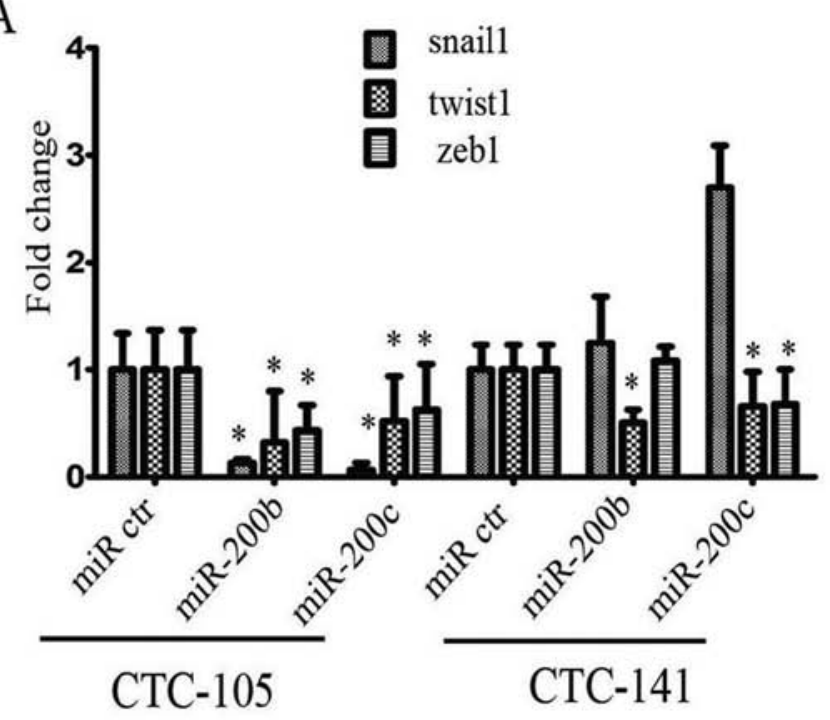

C
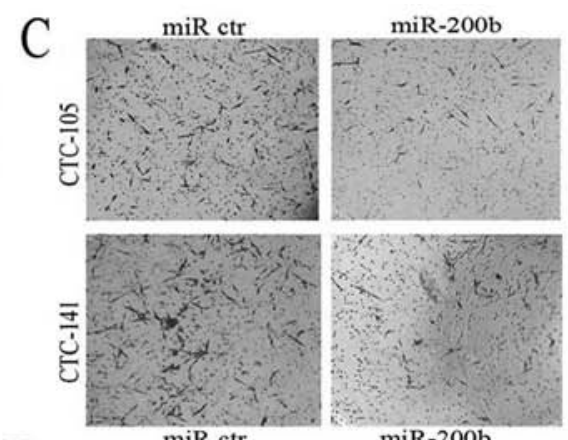

D
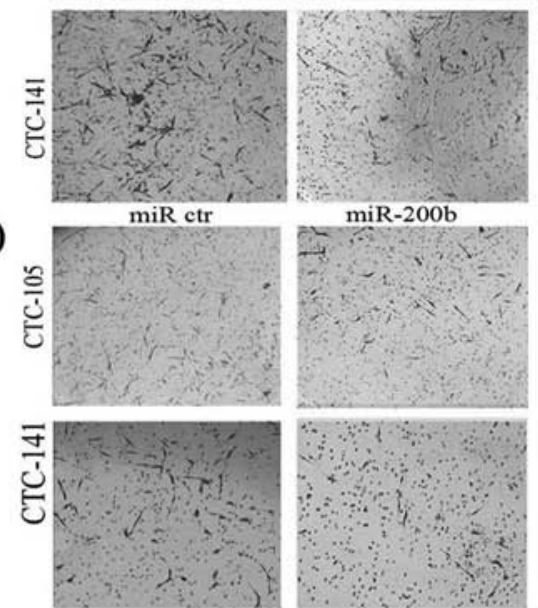

$\mathrm{miR}-200 \mathrm{~b}$
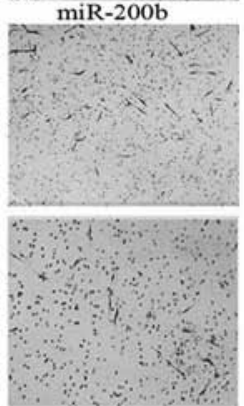

E

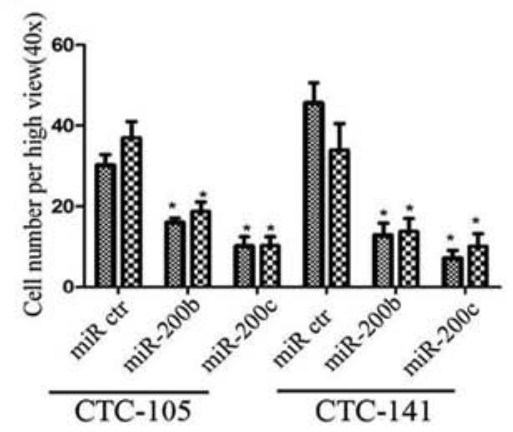

miR-200c
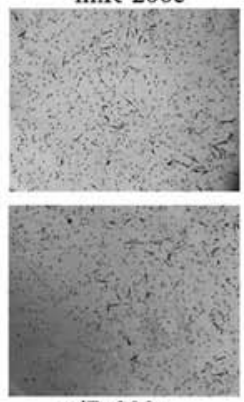

miR-200c

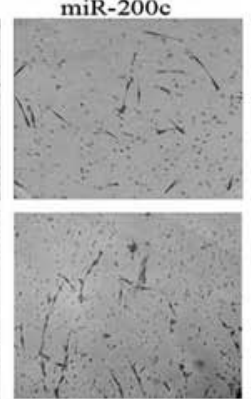

Migration

Invasion
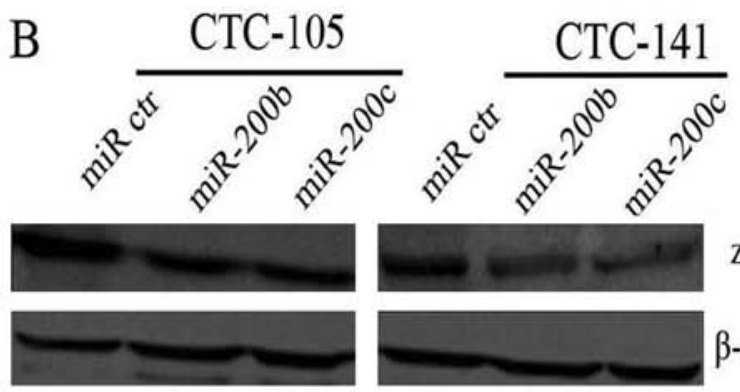

zebl(120 KDa)

$\beta$-actin(42 KDa)

Figure 5. EMT related transcriptors and property of migration and invasion of CTCs were calculated after transfection of miR-200s. (A) Fold change of twist1, snaill, zeb1 mRNA levels were calculated after transfection of miR-200s into gastric CTCs by quantitative real-time PCR, relative expression of the genes was calculated using the method $2^{-\Delta \Delta C T}$ ("P $<0.05$, vs miR ctr). (B) Zeb1 protein level was determined by western blotting after transfection of miR-200s into gastric CTCs. (C) Representative results of transwell migration assays of CTCs scanned through a microscope (x10 original magnification). (D) Representative results of transwell invasion assays of CTCs scanned through microscope (x10 original magnification). (E) Number of cells per high view that migrated through the transwell membrane. Results are presented as means of 15 high power views (x40 original magnification). A representative experiment in triplicate, along with standard errors is shown. One-way analysis of variance (ANOVA) was used to analyze differences among groups and the LSD multiple comparison test was used to identify differences among different groups ( $\mathrm{P}<0.05$, vs miR ctr). miR ctr, CTCs trasfected with negative control miRNA.

regulation of cell migration and invasion. To test whether the cell migration and invasion potential of gastric CTCs transfected with miR-200b or c were inhibited, transwell assay was performed. As shown in Fig. 5C-E, compared with negative control miRNA, ectopic expression of miR-200b or c inhibited migration and invasion potential $(\mathrm{P}<0.05$ for all comparisons, Fig. 5E). Based on the above, miR-200s play a negative role in invasion and metastasis of human gastric CTCs. These results indicated that miR-200b and c inhibited migration and invasion potential in gastric CTCs.

Inhibition of p-Akt increased expression of MicroRNA-200s in gastric CTCs. Virtakoivu et al found that inhibited Akt2 could induce overexpression of miR-200s and then regulated invasion and migration of prostate cancer cells (13). To detect the Akt kinase activation on the expression of miR-200s, PI3K inhibitor $10 \mu \mathrm{M}$ LY294002 was added to CTCs and then expression of miR-200s were detected by real-time PCR. As shown in Fig. 6, the PI3K inhibitor LY294002 inhibited phosphorylation of $\mathrm{AKt}$, and increased the expression of miR-200a, b and c both in CTC-105 and CTC-141 cells. This effect suggests that the function of miR-200s in the gastric CTCs is likely, at least in part, regulated by p-AKt signaling pathway.

\section{Discussion}

Epithelial-mesenchymal (EM) transition (EMT) is a process where cells lose their epithelial properties and obtain mesenchymal properties. EMT has the ability to enhance tumor cell invasion and metastasis (7). Usually in the process, suppressed expression of E-cadherin can induce increased expression of $\mathrm{N}$-cadherin, which is closely related to tumor invasion. Except for invasion, EMT was also reported in the process of drug resistance through multisignal transduction. For example, Ren et al found that inhibition of ZEB1 reverses EMT and chemoresistance in docetaxel-resistant human lung adenocarcinoma cell line (14), Rosano et al found that activated endothelin A 


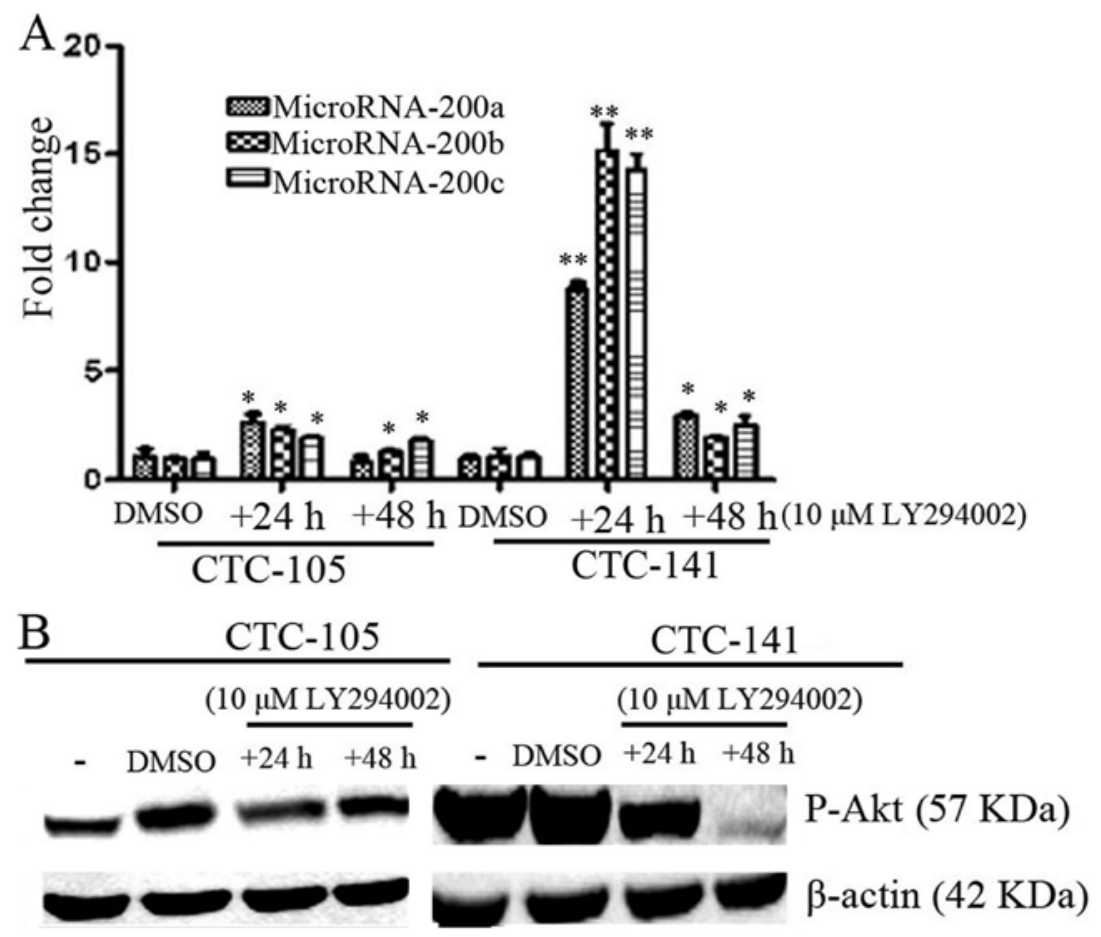

Figure 6. Effect of p-Akt on expression of miR-200s. (A) miR-200s were calculated after addition of PI3K inhibitor LY294002 24 and 48 h by quantitative real-time PCR. Fold changes were calculated using the method $2^{-\Delta \Delta C T}$. (B) p-Akt protein level was detected in CTCs after addition of PI3K inhibitor LY294002 by western blotting. DMSO, as control. Data are presented as means \pm SD from three independent experiments, one of three similar experiments is shown, $($ ( $\mathrm{P}<0.05$, vs DMSO).

receptor pathway enables cells to acquire EMT, thus contributing to chemotherapy resistance to cisplatinum and taxol in ovarian cancer cell lines (15).

In our study, CTCs in human gastric cancer patients showed remarkable EMT process compared with human gastric cancer cell lines SGC-7901 and MKN-45. In the process of dissemination to peripheral blood, cells escaped the primary tumor and acquired mesenchymal composition. $\mathrm{N}$-cadherin, vimentin promoted cell migration and invasion. The process of EMT occur in many tumors, such as in breast cancer $(16)$, cervical cancer $(17,18)$, colorectal cancer (19). EMT was regulated by many pathways involving tumor invasion and metastasis. A study showed that circulating breast tumor cells occurred in different stages of breast cancer patients, and found that patients exhibited dynamic EMT changes in CTCs (11).Others found that breast CTCs co-expressed stem cell-related markers and EMT associated markers $(20,21)$.

Interestingly, our results found that miR-200s were downregulated by activated phosphor-Akt, and the signaling pathway was involved in EMT, migration and invasion of human gastric CTCs. The following signaling pathways involved in EMT process have been reported: notch3-zeb-EMT mediated differentiation of esophageal cancer cells (22), activation of Akt-GSK3 $\beta$-Snail-EMT pathway was involved in the phenomenon of gefitinib resistance in lung cancer (23). TGF- $\beta$-p38 MAPK-EMT (24). Akt-HSF-1-Slug participated in EMT in HER2positive breast cancer (25). AKT-snail-EMT was involved in migration of esophageal squamous cell carcinoma (26); $\beta$-catenin/tcf-zeb1 influenced EMT thereby affecting tumor cell invasion (27). LPS-NF-KB-snail-EMT (28); miR-200a-Wnt/ $\beta$-catenin-zeb-EMT $(29,30)$; activation of src induced EMT (31). TNF $\alpha$-AKT/GSK-3 $\beta$-Snail mediated EMT in colon cancer cells (32). Wnt3-Wnt/ $\beta$-catenin signaling pathway induced EMT-like phenotype and in turn affected the trastuzumab resistance in HER2-positive breast cancer cells (33). mTOR complex 1 affects epithelial type through the opposite regulation of ZEB1/ZEB2 and miR-200b and miR-200c (34). Our study found that, p-Akt-miR200s-zeb1/ twist1 mediated EMT thus affected migration and invasion of human gastric circulating tumor cells. Whether mTOR was involved in the EMT process was not assessed. In conclusion, combined with previous studies, multiple signaling pathways were involved in EMT or EMT-related transcription factors by affecting snail/slug/twist/zeb1/2, thereby affecting the biological behavior in different cells, including cell differentiation, migration and invasion and metastasis, and drug resistance.

Our study had several limitations. Four cases of CTCs were detected, more cases should be involved in the study. Another limitation is that the downstream molecular of $\mathrm{p}-\mathrm{Akt}$ should be detected in order to investigate which pathway was involved in downregulation of miR-200s.

\section{Acknowledgements}

We greatly appreciate the financial support from the National Basic Research Program of China (973 Program, 2011CB935800) (http://www.973.gov.cn/English/Index.aspx) and the Program for Changjiang Scholars and Innovative Research Team in University (PCSIRT, grant no. IRT1272) of China. 


\section{References}

1. Ferlay J, Soerjomataram I, Ervik M, Dikshit R, Eser S, Mathers C, Rebelo M, Parkin DM, Forman D, Bray, F. GLOBOCAN 2012 v1.0, Cancer Incidence and Mortality Worldwide: IARC CancerBase No. 11 [Internet].Lyon, France: International Agency for Research on Cancer; 2013. http://globocan.iarc.fr. Accessed December 18, 2013.

2. Bidard FC, Mathiot C, Delaloge S, et al: Single circulating tumor cell detection and overall survival in nonmetastatic breast cancer. Ann Oncol 21: 729-733, 2010.

3. Cristofanilli M, Budd GT, Ellis MJ, et al: Circulating tumor cells, disease progression, and survival in metastatic breast cancer. N Engl J Med 351: 781-791, 2004.

4. Hayes DF, Cristofanilli M, Budd GT, et al: Circulating tumor cells at each follow-up time point during therapy of metastatic breast cancer patients predict progression-free and overall survival. Clin Cancer Res 12: 4218-4224, 2006.

5. Pantel $\mathrm{K}$ and Alix-Panabières C: Circulating tumour cells in cancer patients: challenges and perspectives. Trends Mol Med 16: 398-406, 2010.

6. Chambers AF, Groom AC and MacDonald IC: Dissemination and growth of cancer cells in metastatic sites. Nat Rev Cancer 2 : 563-572, 2002

7. Thiery JP: Epithelial-mesenchymal transitions in tumour progression. Nat Rev Cancer 2: 442-454, 2002.

8. Gheldof A and Berx G: Cadherins and epithelial-to-mesenchymal transition. Prog Mol Biol Transl Sci 116: 317-336, 2013.

9. Martinez-Alvarez C, Blanco MJ, Perez R, et al: Snail family members and cell survival in physiological and pathological cleft palates. Dev Biol 265: 207-218, 2004.

10. Yu W, Kamara H and Svoboda KK: The role of twist during palate development. Dev Dyn 237: 2716-2725, 2008.

11. Yu M, Bardia A, Wittner BS, et al: Circulating breast tumor cells exhibit dynamic changes in epithelial and mesenchymal composition. Science 339: 580-584, 2013.

12. Williams LV, Veliceasa D, Vinokour E and Volpert OV: miR-200b inhibits prostate cancer EMT, growth and metastasis. PLoS One 8: e83991, 2013.

13. Virtakoivu R, Pellinen T, Rantala JK, Perälä M and Ivaska J: Distinct roles of AKT isoforms in regulating $\beta 1$-integrin activity, migration, andinvasion in prostate cancer. Mol Biol Cell 23: 3357-3369, 2012.

14. Ren J, Chen Y, Song H, Chen L and Wang R: Inhibition of ZEB1 reverses EMT and chemoresistance in docetaxel-resistant human lung adenocarcinoma cell line. J Cell Biochem 114: 1395-1403, 2013.

15. Rosano L, Cianfrocca R, Spinella F, et al: Acquisition of chemoresistance and EMT phenotype is linked with activation of the endothelin A receptor pathway in ovarian carcinoma cells. Clin Cancer Res 17: 2350-2360, 2011.

16. Guarino M, Rubino B and Ballabio G: The role of epithelialmesenchymal transition in cancer pathology. Pathology 39 305-318, 2007

17. Gao Q, Liu W, Cai J, Li M, Gao Y, Lin W and Li Z: EphB2 promotes cervical cancer progression by inducing epithelialmesenchymal transition. Hum Pathol 45: 372-381, 2014.

18. Mayer A, Hockel M, Schlischewsky N, Schmidberger H, Horn LC and Vaupel P: Lacking hypoxia-mediated downregulation of E-cadherin in cancers of the uterine cervix. Br J Cancer 108: 402-408, 2013.

19. Pichler M, Ress AL, Winter E, et al: MiR-200a regulates epithelial to mesenchymal transition-related gene expression and determines prognosis in colorectal cancer patients. Br J Cancer 110: 1614-1621, 2014.
20. Raimondi C, Gradilone A, Naso G, et al: Epithelial-mesenchymal transition and stemness features in circulating tumor cells from breast cancer patients. Breast Cancer Res Treat 130: 449-455, 2011.

21. Armstrong AJ, Marengo MS, Oltean S, et al: Circulating tumor cells from patients with advanced prostate and breast cancer display both epithelial and mesenchymal markers. Mol Cancer Res 9: 997-1007, 2011.

22. Ohashi S, Natsuizaka M, Naganuma S, et al: A NOTCH3mediated squamous cell differentiation program limits expansion of EMT-competent cells that express the ZEB transcription factors. Cancer Res 71: 6836-6847, 2011.

23. Maseki S, Ijichi K, Tanaka H, et al: Acquisition of EMT phenotype in the gefitinib-resistant cells of a head and neck squamous cell carcinoma cell line through Akt/GSK-3ß/snail signaling pathway. Br J Cancer 106: 1196-1204, 2012.

24. Wei J, Li Z, Chen W, Ma C, Zhan F, Wu W and Peng Y: AEG-1 participates in TGF-beta1-induced EMT through p38 MAPK activation. Cell Biol Int 37: 1016-1021, 2013.

25. Carpenter RL, Paw I, Dewhirst MW and Lo HW: Akt phosphorylates and activates HSF-1 independent of heat shock, leading to Slug overexpression and epithelial-mesenchymal transition (EMT) of HER2-overexpressing breast cancer cells. Oncogene: Jan 27, 2014 (Epub ahead of print). doi: 10.1038/onc.2013.582.

26. Okui G, Tobiume K, Rizqiawan A, et al: AKT primes snailinduced EMT concomitantly with the collective migration of squamous cell carcinoma cells. J Cell Biochem 114: 2039-2049, 2013.

27. Sanchez-Tillo E, de Barrios O, Siles L, Cuatrecasas M, Castells A and Postigo A: beta-catenin/TCF4 complex induces the epithelial-to-mesenchymal transition (EMT)-activator ZEB1 to regulate tumor invasiveness. Proc Natl Acad Sci USA 108: 19204-19209, 2011.

28. Huang T, Chen $Z$ and Fang L: Curcumin inhibits LPS-induced EMT through downregulation of NF-kappaB-Snail signaling in breast cancer cells. Oncol Rep 29: 117-124, 2013.

29. Liu J, Ruan B, You N, et al: Downregulation of miR-200a induces EMT phenotypes and CSC-like signatures through targeting the $\beta$-catenin pathway in hepatic oval cells. PLoS One 8: e79409, 2013.

30. Cong N, Du P, Zhang A, et al: Downregulated microRNA-200a promotes EMT and tumor growth through the wnt/beta-catenin pathway by targeting the E-cadherin repressors ZEB1/ZEB2 in gastric adenocarcinoma. Oncol Rep 29: 1579-1587, 2013.

31. Zhao Y, Li X, Sun X, Zhang Y and Ren H: EMT phenotype is induced by increased Src kinase activity via Src-mediated caspase-8 phosphorylation. Cell Physiol Biochem 29: 341-352, 2012.

32. Wang H, Wang HS, Zhou BH, et al: Epithelial-mesenchymal transition (EMT) induced by TNF-alpha requires AKT/GSK-3betamediated stabilization of snail in colorectal cancer. PLoS One 8: e56664, 2013.

33. Wu Y, Ginther C, Kim J, Mosher N, Chung S, Slamon D and Vadgama JV: Expression of Wnt 3 activates Wnt/beta-catenin pathway and promotes EMT-like phenotype in trastuzumabresistant HER2-overexpressing breast cancer cells. Mol Cancer Res 10: 1597-1606, 2012.

34. Mikaelian I, Malek M, Gadet R, et al: Genetic and pharmacologic inhibition of mTORC1 promotes EMT by a TGF- $\beta$-independent mechanism. Cancer Res 73: 6621-6631, 2013. 